\title{
Sterilization of Scaffolds of Calcium Phosphates and Bacterial Cellulose for their Use in Tissue Regeneration
}

\author{
Erick Mark Francisco $^{1}$ (D), Jacqueline de Oliveira Zoccolotti ${ }^{2}{ }^{(D)}$, Olinka Tiomno Tiomnova ${ }^{3}$ (D), Angel \\ Gustavo Tolaba (D) 4 , Jorge Enrique Rodriguez Chanfrau 1,*iD, Janaina Habib Jorge 2(D), Pierre \\ Basmaji 5 (D), Antonio Carlos Guastaldi ${ }^{1}$ (D) \\ 1 Department of Physical Chemistry, Institute of Chemistry, São Paulo State University (UNESP), Araraquara, Brazil \\ 2 Department of Dental Materials and Prostheses, Araraquara Dental School, São Paulo State University (UNESP), \\ Araraquara, Brazil \\ 3 Center of Engineering and Chemical Research, Havana, CP 10600. Cuba \\ 4 Mathematics Department, Science Faculty. National University of Salta, Salta, Argentina \\ 5 Innovatec's - Biotechnology Research and Development, São Carlos-SP, Brazil \\ * Correspondence: jerodriguez354@gmail.com;
}

Scopus Author ID 7801488065

Received: 17.09.2020; Revised: 7.10.2020; Accepted: 9.10.2020; Published: 12.10.2020

\begin{abstract}
The development of biomaterials for the regeneration of tissues is of great importance, and their demand increases every day, due to the increase of the aging population, the expectation, and quality of life, as well as the increase of the accident rates (traffic and violence). Scaffolds are a threedimensional structure designed to withstand cellular infiltration, growth, and differentiation in order to improve the development and formation of new tissues. Many biomaterials can be used to make these structures, such as bioceramics and biopolymers. However, few studies have been conducted to evaluate its microbiological contamination and the influence of sterilization methods may have on the structure and properties. The objective of this work was to evaluate different sterilization processes and their influence on the physical-chemical properties of Scaffolds made with calcium phosphate and bacterial cellulose. Three sterilization techniques (steam sterilization, ultraviolet radiation sterilization, and microwave sterilization) were evaluated. The results showed that steam sterilization was effective in eliminating microorganisms. Microwave sterilization significantly reduced the amount of CFU / $\mathrm{mL}$, while ultraviolet radiation sterilization did not show efficacy. Changes in the physical-chemical properties of the Scaffolds evaluated after sterilization was not observed, except a slight darkening in the samples treated by steam sterilization or treated by microwave sterilization due to the presence of sodium alginate in the formulation. It was concluded that the steam sterilization process was the most effective.
\end{abstract}

Keywords: sterilization process; scaffolds; calcium phosphates; bacterial cellulose; statistical analysis.

(C) 2020 by the authors. This article is an open-access article distributed under the terms and conditions of the Creative Commons Attribution (CC BY) license (https://creativecommons.org/licenses/by/4.0/).

\section{Introduction}

The demand for biomaterials for tissue regeneration increases every day due to factors such as the increasing aging of the population, the expectation and quality of life, and the increase in accidents (traffic and violence) [1-3]. Scaffolds are three-dimensional structures designed to resist cell infiltration, growth, and differentiation in order to enhance the development and formation of new tissues. Many biomaterials are used to make these 
structures, including bioceramics and biopolymers [4-8]. At the same time, many technologies have been studied to make these materials [9-18].

Within the process of making Scaffold for "In vivo" use, a fundamental step should be the sterilization process. Sterilization is a process by which a product is free from contamination by living microorganisms. One of the characteristics that biomaterials must possess is that they must be biodegradable, being materials that become targets of microbiological contamination. When biodegradable structures are sterilized, the chosen sterilization technique must maintain the structural, physical-chemical, mechanical, and biochemical properties of the Scaffolds, ensuring that they will fulfill the intended purposes after sterilization [5]. However, despite the importance of this stage in the process of making Scaffold, few works are reported in the literature in which the influence of sterilization on the properties of Scaffolds is evaluated [5, 19-25]. The objective of this work was to evaluate different sterilization processes and their influence on the physical-chemical properties of Scaffolds made with calcium phosphate and bacterial cellulose.

\section{Materials and Methods}

The elaboration of the scaffolds was carried out by applying the methodology described by Tiomno et al. [26].

\subsection{Study of sterilization of scaffolds.}

Three types of sterilization were evaluated: microwave sterilization, steam sterilization, and ultraviolet radiation sterilization. In all cases, 12 samples were sterilized (10 for biological evaluation and 2 for chemical evaluation).

\subsection{Microwave sterilization.}

The samples were placed inside conventional microwave equipment. The microwave was applied for 5 minutes at a power of 100 Watts [21].

\subsection{Steam sterilization.}

The samples were placed in a vertical autoclave, brand FABBE, model 103. The scaffolds were sterilized under the pressure of 1 atm and a temperature of $121^{\circ} \mathrm{C}$ for 20 minutes [5].

\subsection{Sterilization by ultraviolet irradiation.}

The samples were sterilized under ultraviolet light, brand FLUOR 30W GL GERMICIDA, manufacturer LUCMAT LAMPS. Each side of the sample was left under radiation for 30 minutes [27].

\subsection{Microbiological evaluation.}

The effect of scaffold disinfection methods was evaluated by counting colony-forming units per milliliter (CFU / mL). Four experimental groups were elaborated: control group (CG), microwave sterilization (MW), steam sterilization (SS), and ultraviolet light (UV) sterilization. Ten $\mathrm{mL}$ of sterile Roswell Park Memorial Institute (RPMI) medium, buffered with morpholinepropanesulfonic acid (MOPS) were added in aseptic Falcon type tubes. 
Subsequently, a sample from each group was added, and the tubes were placed in an incubation oven at $37^{\circ} \mathrm{C}$ for 12 hours for cell dispersion present inside the samples and obtaining of the eluates. After 12 hours, the tubes were vortexed for 1 minute. Then, the serial dilution process was carried out in Eppendorf tubes containing $900 \mu \mathrm{L}$ of pure phosphate-buffered saline (PBS) by transferring $100 \mu \mathrm{L}$ aliquots of the original solution. Dilutions of $10^{-1}$ to $10^{-4}$ were obtained. Two $25 \mu \mathrm{L}$ aliquots of the $10^{-3}$ and $10^{-4}$ dilution were dripped into two Petri dishes containing the following media: Sabouraud Dextrose Agar with Chloramphenicol (SDA), Brain and Heart Infusion Agar (BHI), Chromagar and Blood Agar. Then, all plates were incubated at $37^{\circ} \mathrm{C}$ for 48 hours. After this period, the plates were placed on a manual colony counter, and the number of colonies was determined. The numbers of colony-forming units per milliliter (CFU / $\mathrm{mL})$ was calculated according to the following formula: $\mathrm{CFU} / \mathrm{mL}=$ number of colonies $\mathrm{x} 25 / \mathrm{q}$. In this formula, " $n$ " is equivalent to the absolute value of the chosen dilution (from 3 to 4 ) and "q" is equivalent to the quantity, in $\mathrm{mL}$, sown for each dilution in the plates $(0.025 \mathrm{~mL})$. Three different experiments were performed in triplicates $(n=9)$. All results were evaluated statistically, and the significance level of $\alpha=0.05$ was used for inference analyses [28].

\subsection{Statistical analysis.}

With the data obtained by the microbiological evaluation, a statistical analysis was performed using one-way ANOVA. First, an exploratory study of the data and the verification of the necessary hypotheses that must be fulfilled to apply a classic ANOVA was carried out. In case of non-compliance with homoscedasticity, Krukal Wallis' non-parametric alternative was used. The statistical software used to perform the study was INFOSTAT version 2017. All tests were evaluated with significance of $\alpha=5 \%$.

\subsection{Characterization of scaffolds after sterilization.}

To study the influence of the sterilization process on the properties of the Scaffolds, an evaluation using Fourier Transform Infrared Spectroscopy (FTIR) and Scanning Electron Microscopy (SEM), applying the experimental procedures described by Tiomno et al. [18] were carried out. The best sterilization variant was evaluated by means of a mechanical test (compression test). The compressive strength test (Emic DL2000, Tesc Program 384) was performed with a $2 \mathrm{kN}$ Trd 28 load cell, using a crossbar speed of $1 \mathrm{~mm} / \mathrm{min}$, according to ISO 5833 standards.

\section{Results and Discussion}

\subsection{Microbiological evaluation.}

In this study, four types of culture media (SDA, Chromagar, Blood Agar, and BHI) were used to evaluate the growth of microorganisms present in sterile and non-sterile (CG) samples.

In the microbiological evaluation using SDA or Chromagar as a medium, no growth of microorganisms was observed for any of the groups tested. These results would allow us to assume that there is no presence of fungi in the tested samples. On the other hand, Tables 1 and 2 show the results of the microbiological evaluation in Blood Agar and in BHI culture media, respectively, for all samples treated with different sterilization methodologies. In all experiments, the dilution was 1000 and "n" equal to 3. 
The results show that the steam sterilization of the working conditions used in this study eliminated the microbial load in the samples evaluated in both media. This result was expected since the efficiency of this sterilization methodology, and its use in different fields of daily life are known. This methodology has a disadvantage that this process is carried out with water vapor, which can, in some cases, increase the humidity in the evaluated materials, which may affect their properties.

In our specific case, taking into account that the formulation has polymers that have high hygroscopicity, humidity studies were previously carried out before and after sterilization, verifying that in our working conditions and on the scale worked, the sterilization process did not affect quality.

\begin{tabular}{|c|c|c|c|c|}
\hline Experiment & Group & $\begin{array}{c}\text { Number of colonies } \\
\text { (Average / DE) }\end{array}$ & UFC/mL & $\log 10$ \\
\hline \multirow{4}{*}{1} & CG & $125.7 / 20.9$ & 5026666.7 & 6.70 \\
\hline & MW & $14.7 / 3.6$ & 586666.7 & 5.77 \\
\hline & UV & $113.3 / 11.1$ & 4533333.3 & 6.66 \\
\hline & SS & 0.0 & 0.0 & 0.00 \\
\hline \multirow{4}{*}{2} & CG & $122.7 / 20.9$ & 4906666.7 & 6.69 \\
\hline & MW & $22.0 / 4.7$ & 880000.0 & 5.94 \\
\hline & $\mathbf{U V}$ & $116.0 / 3.3$ & 4640000.0 & 6.67 \\
\hline & SS & 0.0 & 0.0 & 0.0 \\
\hline \multirow{4}{*}{3} & CG & $167.0 / 23.3$ & 6680000.0 & 6.82 \\
\hline & MW & $23.7 / 8.2$ & 946666.7 & 5.98 \\
\hline & UV & $132.0 / 19.3$ & 5280000.0 & 6.72 \\
\hline & SS & 0.0 & 0.0 & 0.0 \\
\hline
\end{tabular}

Table 2. Microbiological results in BHI culture media.

\begin{tabular}{c|c|c|c|c}
\multirow{2}{*}{ Experiment } & Group & $\begin{array}{c}\text { Number of colonies } \\
\text { (Average / DE) }\end{array}$ & UFC/mL & Log10 \\
\hline \multirow{4}{*}{$\mathbf{1}$} & CG & $299.3 / 0.9$ & 11973333.3 & 7.08 \\
\cline { 2 - 5 } & MW & $20.7 / 5.6$ & 826666.7 & 5.92 \\
\cline { 2 - 5 } & UV & $300.0 / 0.7$ & 12000000.0 & 7.08 \\
\cline { 2 - 5 } & SS & 0.0 & 0.0 & 0.00 \\
\hline \multirow{4}{*}{$\mathbf{N}$} & CG & $299.7 / 0.4$ & 11986666.7 & 7.08 \\
\cline { 2 - 5 } & MW & $17.3 / 3.6$ & 693333.3 & 5.84 \\
\cline { 2 - 5 } & UV & $299.7 / 1.1$ & 11986666.7 & 7.08 \\
\cline { 2 - 5 } & SS & 0.0 & 0.0 & 0.0 \\
\hline \multirow{3}{*}{3} & CG & $299.3 / 0.9$ & 11973333.3 & 7.08 \\
\cline { 2 - 5 } & MW & $21.0 / 6.0$ & 840000.0 & 5.92 \\
\cline { 2 - 5 } & UV & $299.3 / 0.4$ & 11973333.3 & 7.08 \\
\cline { 2 - 5 } & SS & 0.0 & 0.0 & 0.0
\end{tabular}

In the case of the other two sterilization methods studied, it was observed that, when applying microwave sterilization (MW), the microbial load content had a reduction of 1 log in the two studied media, and this did not occur when the ultraviolet sterilization (UV) was applied.

\subsection{Statistical analysis.}

Starting from the experimental data, the statistical analysis was carried out. In this case, only the MW and UV groups were evaluated, since the SS treatment was effective in the total elimination of the microorganisms present.

First, the three experiments were compared statistically by ANOVA for each of the groups studied, in order to assess whether there were significant differences between the results of each experiment. Table 3 shows the results of the evaluation, noting that in none of the cases, 
there was a significant difference between the experimental replicates, it could be said that the established analytical procedure is reproducible.

Table 3. Results of the statistical comparison between the replicas of each group.

\begin{tabular}{c|c|c|c|c} 
Culture media & Group & $\mathbf{n}$ & Average/DE & $\mathbf{p}$ \\
\hline \multirow{3}{*}{ Blood Agar } & CG & 3 & $138.5 / 1.9$ & 0.480 \\
\cline { 2 - 5 } & MW & 3 & $20.1 / 3.6$ & 0.319 \\
\cline { 2 - 5 } & UV & 3 & $120.4 / 7.7$ & 0.952 \\
\hline \multirow{3}{*}{ BHI } & CG & 3 & $299.4 / 0.2$ & 0.973 \\
\cline { 2 - 5 } & MW & 3 & $19.7 / 1.6$ & 0.077 \\
\cline { 2 - 5 } & UV & 3 & $299.7 / 0.2$ & 0.872
\end{tabular}

Based on these results, the study carried out for each sterilization group was considered as a single experiment, assuming $\mathrm{n}$ as 9 . The results of the statistical study are shown in Table 4.

Table 4. Average values of the analysis of the samples treated with different sterilization techniques.

\begin{tabular}{c|c|c|c} 
Culture media & Group & n & Average/DE \\
\hline \multirow{3}{*}{ Blood Agar } & CG & 9 & $138.4 / 2.4$ \\
\cline { 2 - 4 } & MW & 9 & $20.1 / 5.7$ \\
\cline { 2 - 4 } & UV & 9 & $120.4 / 7.8$ \\
\hline \multirow{3}{*}{ BHI } & CG & 9 & $299.4 / 0.7$ \\
\cline { 2 - 4 } & MW & 9 & $19.7 / 4.6$ \\
\cline { 2 - 4 } & UV & 9 & $299.7 / 0.8$
\end{tabular}

The statistical comparison study for samples evaluated on Blood Agar medium showed that the means of samples sterilized by microwaves are different from the means of samples sterilized by UV and control samples (Table 4). There is an agreement in the normality of the residual of the response variables verified by means of the normal quantile graph (Q-Q Plot) that there is a strong correlation $(\mathrm{r}=0.971)$ with the straight of the normal quantiles. While, when applying the Shapiro-Wilks test, it appears that the p-value (0.5159) is greater than the $\alpha$ value (0.05); therefore, one can assume that the response variables are normal, that is, there is normality.

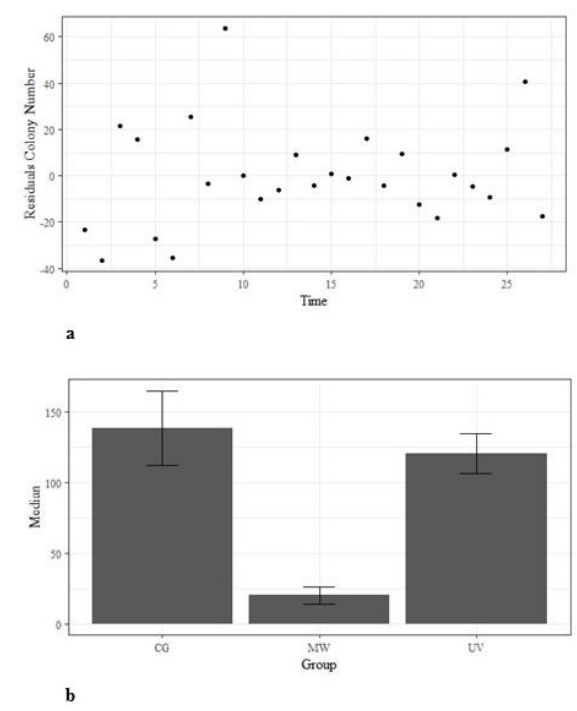

Figure 1. Scatter diagram (a) and comparison graph of medians using the Kruskal Wallis test (b) in Ágar Sangue medium.

On the other hand, the scatter plot of the residuals of the response variables according to the order in which the data were taken shows a random behavior (Figure 1a), which guarantees the independence of the data. When analyzing homoscedasticity, it is confirmed 
that when applying the Levene test, a p-value (0.0023) lower than the value of $\alpha(0.05)$ is obtained, which allows the null hypothesis Ho to be rejected, being able to assume that the variances of the subpopulations are not homogeneous. Therefore there is no homoscedasticity.

When this occurs, the Kruskal Wallis non-parametric test and the non-parametric contrast test (post-hoc) are performed. The results showed that the p-value (0.0001) is less than the $\alpha$ value (0.05), so there are significant differences in the average number of colonies after the application of the different sterilization treatments (Figure 1b). The contrast test showed a significant difference between the control group and the sterile samples applying MW. For the case of samples treated using UV methodology, no significant difference is observed.

The statistical comparison study for the samples evaluated in BHI culture medium, as in the previous case, showed that the means of the samples sterilized by microwaves are different from the means of the samples sterilized by UV and the control samples (Table 4). However, contrary to what happened in the case of studies carried out on a blood agar medium, there was a non-conformity in the normality of the residue of the variables responses verified through the normal quantile graph (QQ Plot) that there is no strong correlation $(r=0.861)$ with the straight line of normal quantiles. Considering that, when applying the Shapiro-Wilks test, it appears that the p-value $(0.0001)$ is less than the $\alpha$ value $(0.05)$; therefore, it can be assumed that the response variables are not normal, that is, there is no normality.

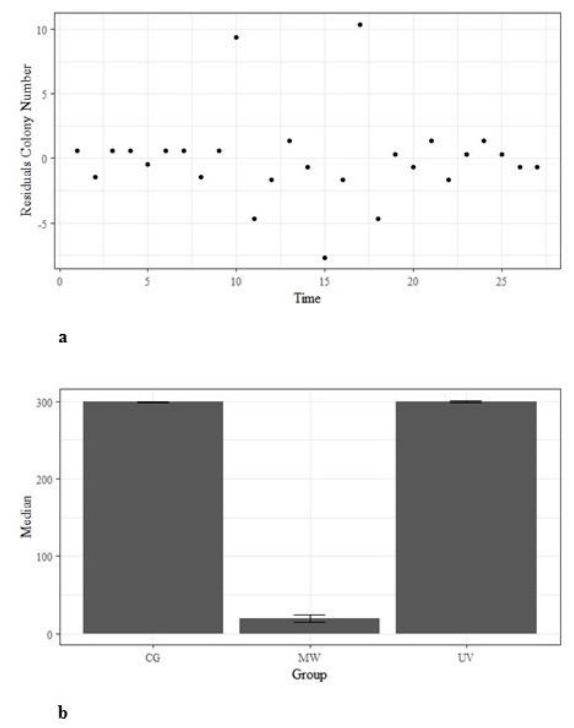

Figure 2. Scatter diagram (a) and comparison graph of medians using the Kruskal Wallis test (b) in BHI medium.

The scatter plot of the residuals of the response variables according to the order in which the data were obtained is not completely random, observing a random trend for a part of the data (Figure 2a). But, taking into account that the experiment is planned in such a way that each sample unit receives only one treatment, we can say that there is independence in the data evaluated. When analyzing homoscedasticity, it is confirmed that when applying the Levene test, a p-value (0.0002) is obtained that is lower than the value of $\alpha(0.05)$, which allows rejecting the null hypothesis Ho, which indicates that the variances of the subpopulations are not homogeneous; therefore there is no homoscedasticity. As homoscedasticity and homogeneity were not satisfied, the Kruskal Wallis non-parametric test and the non-parametric contrast test (post-hoc) were performed. The results showed that the p-value (0.0001) is less than the $\alpha$ value (0.05); therefore, there are significant differences in the average number of 
colonies after the application of the different sterilization treatments (Figure 2b). The contrast test showed a significant difference between the control group and the sterile samples applying MW. In the case of samples treated using UV methodology, no significant difference is observed.

In general, it has been shown that treatment with MW guarantees a significant decrease in the microbial load. Meanwhile, UV treatment does not guarantee a decrease of microorganisms.

\subsection{Characterization of scaffolds after sterilization.}

After the sterilization process, the samples were analyzed by Infrared Spectroscopy with Fourier Transform (FTIR) and Scanning Electron Microscopy (SEM) to determine whether the sterilization process affected the physical-chemical composition of the samples. Figure 3 shows the results of the analysis of the samples by FTIR Spectroscopy. No substantial changes were observed in the spectra of the treated samples, except in the case of the sample sterilized by UV irradiation, where the intensity of the peaks is greater. On the other hand, Figure 4 shows the images obtained by electron microscopy of the treated samples, noting changes in the morphology of the samples.

In general, a slight change in color of the steam or microwave sterilized samples was observed. In our opinion, it is due to the presence of sodium alginate, which tends to darken in the presence of heat.

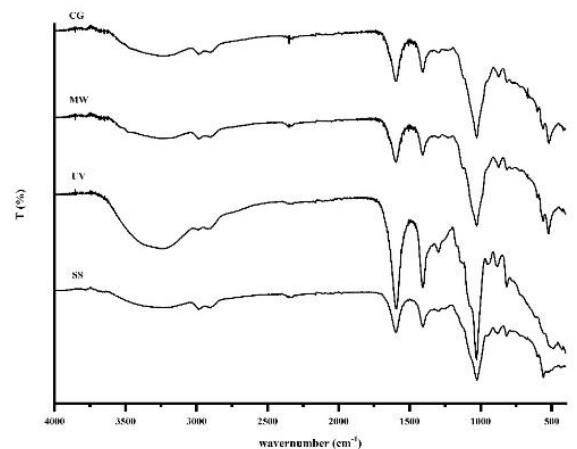

Figure 3. Results of the FTIR evaluation of the samples treated with different sterilization processes.

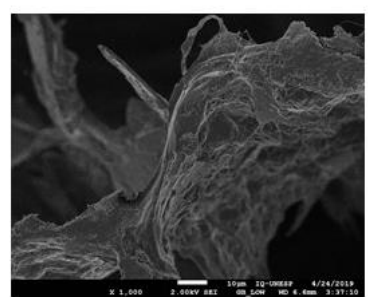

a

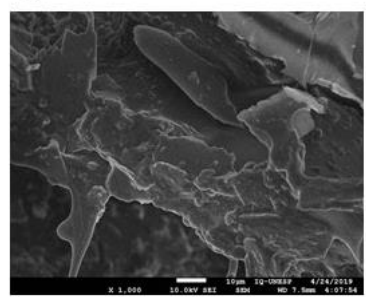

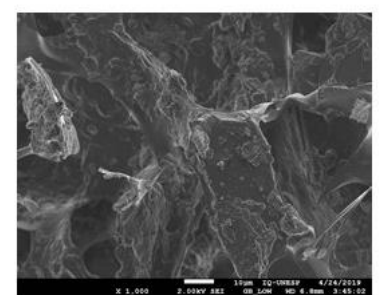

b

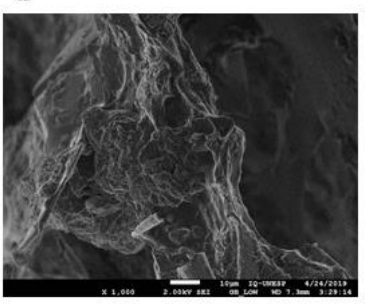

d

Figure 4. Results of the evaluation by scanning electron microscopy (1000X) of the samples treated with different sterilization processes. (a: CG; b: MW; c: UV and d: SS). 


\subsection{Mechanical test.}

The results of the mechanical test on the steam sterilized samples showed that during the test, the samples undergo a deformation process, without breaking them. This phenomenon can be attributed to the presence of polymers (sodium alginate and bacterial cellulose) in the formulation. It has been reported in the literature that during the application of a load at a constant speed, the present polymers respond efficiently to the applied tension, absorbing energy and dissipating it without fracturing the part [29].

The results showed that the tensile strength was $\sigma=66.74 \pm 0.09 \mathrm{MPa}$ and $\sigma=66.76 \pm$ 0.15 MPa for the CG samples and the SS samples, respectively. These results indicate that the steam sterilization process, under the conditions evaluated in this work, does not affect the mechanical properties of the Scaffolds studied.

\section{Conclusions}

Three Scaffolds sterilization processes were studied. The microbiological analyzes were carried out in four culture media, showing that there was no fungal growth in the studied variants. The most effective methodology was steam sterilization. On the other hand, microwave sterilization causes a logarithmic reduction equal to 1 under the conditions studied, while ultraviolet light sterilization did not show efficacy. Changes in the physical-chemical properties of the Scaffolds evaluated after sterilization was not observed, except a slight darkening in the samples treated by steam sterilization or treated by microwave sterilization due to the presence of sodium alginate in the formulation. This aspect must be taken into consideration when applying these processes.

\section{Funding}

This research was funded by the Coordination for the Improvement of Higher Education Personnel (CAPES) (CAPES-MES project 186/13) and The São Paulo Research Foundation FAPESP (project 2017 / 15487-3).

\section{Acknowledgments}

The authors thank Products Biotechnological LTDA, São Carlos - São Paulo, Brazil, for the support needed to carry out this work. To Thales A. Pelizaro for his help in sterilizing the samples. To the Post-Graduate Program in Biomaterials and Bioprocess Engineering (Master's Degree Profissional) of the Faculty of Pharmaceutical Sciences, UNESP. Araraquara. Brazil.

\section{Conflicts of Interest}

The authors declare no conflict of interest.

\section{References}

1. McNamara, S.L.; Rnjak-Kovacina, J.; Schmidt, D.F.; Lo, T.J.; Kaplan, D.L. Silk as a biocohesive sacrificial binder in the fabrication of hydroxyapatite load bearing scaffolds. Biomaterials 2014, 35, 6941-6953, https://doi.org/10.1016/j.biomaterials.2014.05.013.

2. Golchin, A.; Farahany, T.Z. Biological Products: Cellular Therapy and FDA Approved Products. Stem Cell Reviews and Reports 2019, 15, 166-175, https://doi.org/10.1007/s12015-018-9866-1.

3. Cuervo-Lozano, C.E.; Soto-Domínguez, A.; Saucedo-Cárdenas, O. Osteogenesis induced by a threedimensional bioimplant composed of demineralised bone matrix, collagen, hydroxyapatite, and bone 
marrow-derived cells in massive bone defects: An experimental study. Tissue Cell 2018, 50, 69-78, https://doi.org/10.1016/j.tice.2017.12.005.

4. Chen, Q.; Zhu, C.; Thouas, G.A. Progress and challenges in biomaterials used for bone tissue engineering: bioactive glasses and elastomeric composites. Prog Biomater. 2012, 1, https://doi.org/10.1186/2194-0517$1-2$.

5. Dai, Z.; Ronholm, J.; Tian, Y.; Sethi, B.; Cao, X. Sterilization techniques for biodegradable scaffolds in tissue engineering applications. Journal of Tissue Engineering 2016, 7 , https://doi.org/10.1177/2041731416648810.

6. Yu, W.; Zhao, H.; Ding, Z. In vitro and in vivo evaluation of $\mathrm{MgF}_{2}$ coated $\mathrm{AZ3} 1$ magnesium alloy porous scaffolds for bone regeneration. Colloids Surf B Biointerfaces 2017, 149, 330-340, https://doi.org/10.1016/j.colsurfb.2016.10.037.

7. Gautam, S.; Dinda, A.K.; Mishra, N.C. Fabrication and characterization of PCL/gelatin composite nanofibrous scaffold for tissue engineering applications by electrospinning method. Materials Science and Engineering: C 2013, 33, 1228-1235, https://doi.org/10.1016/j.msec.2012.12.015.

8. Golchin, A.; Farahany, T.Z. Biological Products: Cellular Therapy and FDA Approved Products. Stem Cell Rev Rep. 2019; 15(2):166-175. https://doi.org/10.1007/s12015-018-9866-1

9. Piazza, R.D.; Pelizaro, T.A.G.; Rodriguez-Chanfrau, J.E.; La Serna, A.A.; Veranes-Pantoja, Y.; Guastaldi, A.C. Calcium phosphates nanoparticles: The effect of freeze-drying on particle size reduction. Materials Chemistry and Physics 2020, 239, https://doi.org/10.1016/j.matchemphys.2019.122004.

10. Rodriguez-Chanfrau, J.E.; Garcia Pelizaro, T.A.; da Silva, R.M.; Tolaba, A.G.; Pizoni, E.; Veranes-Pantoja, Y.; Guastaldi, A.C. Synthesis by Wet Chemical Method of Different Phases of Apatites Applying Ultrasound. Journal of Bionanoscience 2018, 12, 134-141, https://doi.org/10.1166/jbns.2018.1502.

11. Garcia Pelizaro, T.A.; Tolaba, A.G.; Rodriguez-Chanfrau, J.E.; Veranes-Pantoja, Y.; Guastaldi, A.C. Influence of the Application of Ultrasound During the Synthesis of Calcium Phosphates. Journal of Bionanoscience 2018, 12, 733-738, https://doi.org/10.1166/jbns.2018.1585.

12. Rodriguez Chanfrau, J.E.; Veranes Pantoja, Y.; Guastaldi, A.C. Ultrasonic application and spray drying during amorphous calcium phosphate synthesis. Letters in Applied NanoBioScience 2019, 8, 711-714, https://doi.org/10.33263/LIANBS84.711714.

13. Rodriguez Chanfrau, J.E.; Tolaba, A.G.; Garcia Pelizaro, T.A.; Veranes Pantoja, Y.; Guastaldi, A.C. Evaluation of the Behavior in Water of a Biomaterial Composed of Different Phases of Calcium Phosphate Letters in Applied NanoBioScience 2020, 9, 15131520.

14. Rodriguez-Chanfrau, J.E.; Veranes-Pantoja, Y.; Basmaji, P.; Guastaldi, A.C. Influence of the reaction time during the treatment of bacterial cellulose with sulfuric acid solution. Biointerface Research in Applied Chemistry 2019, 9, 4301-4304.

15. Basu, P.; Saha, N.; Alexandrova, R.; Saha, P. Calcium Phosphate Incorporated Bacterial CellulosePolyvinylpyrrolidone Based Hydrogel Scaffold: Structural Property and Cell Viability Study for Bone Regeneration Application. Polymers (Basel) 2019, 11, https://doi.org/10.3390/polym11111821.

16. Abdul Khalil, H.P.S.; Jummaat, F.; Bashir Yahya, E.; Olaiya, N.G.; Adnan, A.S.; Abdat, M.; Nasir, N.A.M.; Sukari Halim, A.; Seeta Uthaya Kumar, U.; Bairwan, Rahul.; Suriani, A.B. A Review on Micro- to Nanocellulose Biopolymer Scaffold Forming for Tissue Engineering Applications. Polymers 2020, 12, https://doi.org/10.3390/polym12092043.

17. Basu, P.; Saha, N.; Saha, P. Swelling and rheological study of calcium phosphate filled bacterial cellulosebased hydrogel scaffold. Applied Polymer 2020, 137, https://doi.org/10.1002/app.48522.

18. Hua, B.; Meng, Z.D.; Zhang, Y.Q.; Ye, L.Y.; Wang, C.J.; Guo, W.C. Sr-HA scaffolds fabricated by SPS technology promote the repair of segmental bone defects. Tissue and Cell 2020, 66, https://doi.org/10.1016/j.tice.2020.101386.

19. Qiu, Q.Q.; Sun, W.; Connor, J. Sterilization of Biomaterials of Synthetic and Biological Origin. Comprehensive Biomaterials 2011, 4, 127-144, https://doi.org/10.1016/B978-0-08-055294-1.00248-8.

20. Uchiyama, K.; Ujihira, M.; Mabuchi, K.; Takahira, N.; Komiya, K.; Itoman, M. Development of heating method by microwave for sterilization of bone allografts. J Orthop Sci. 2005, 10, 77-83, https://doi.org/10.1007/s00776-004-0857-5.

21. Kang, H.W.; Tabata, Y.; Ikada, Y. Fabrication of porous gelatin scaffolds for tissue engineering. Biomaterials 1999, 20, 1339-1344, https://doi.org/10.1016/s0142-9612(99)00036-8.

22. Guimarães Pellissari, C.V.; Vergani, C.E.; Longo, E.; Pavarina, A.C.; Volpato Sanitá, P.; Siqueira, W.L.; Habib Jorge, J. In Vitro Toxic Effect of Biomaterials Coated with Silver Tungstate or Silver Molybdate Microcrystals, Journal of Nanomaterials 2020, 2020, https://doi.org/10.1155/2020/2971827.

23. Zoccolotti, J.O.; Suzuki, R.B.; Rinaldi, T.B.; Pellissari, C.V.G.; Sanitá, P.V.; Jorge, J.H. Physical properties of artificial teeth after immersion in liquid disinfectant soaps. Am J Dent. 2019, 32, 14-20.

24. Rigolin, M.S.M.; Barbugli, P.A.; Jorge, J.H.; Reis, M.R.D.; Adabo, G.L.; Casemiro, L.A.; Martins, C.H.G.; de Lima, O.J.; Mollo Junior, F.A. Effect of the aging of titanium and zirconia abutment surfaces on the viability, adhesion, and proliferation of cells and the adhesion of microorganisms. J Prosthet Dent. 2019, 122, 564.e1-564.e10, https://doi.org/10.1016/j.prosdent.2019.08.027. 
25. Moradi, L.; Mohammadi Jobania, B.; Jafarnezhad-Ansariha, F.; Ghorbani, F.; Esmaeil-Pour, R.; Majidi Zolbina, M.; Kajbafzadeh, A.M. Evaluation of different sterilization methods for decellularized kidney tissue. Tissue and Cell 2020, 66, https://doi.org/10.1016/j.tice.2020.101396.

26. Tiomno-Tiomnova, O.; Coelho, F.; Garcia Pelizaro, T.A.; Rodriguez-Chanfrau, J.E.; Capote, T.S.; Basmaji, P.; Veranes-Pantoja, Y.; Guastaldi, A.C. Preparation of Scaffolds of Amorphous Calcium Phosphate and Bacterial Cellulose for Use in Tissue Regeneration by Freeze-Drying Process. Biointerface Research in Applied Chemistry 2021, 11, 7357-7367, https://doi.org/10.33263/BRIAC111.73577367.

27. Yixiang, D.; Yong, T.; Liao, S.; Chan, C.K.; Ramakrishna, S. Degradation of electrospun nanofiber scaffold by short wave length ultraviolet radiation treatment and its potential applications in tissue engineering. Tissue Eng Part A 2008, 14, 1321-1329, https://doi.org/10.1089/ten.tea.2007.0395.

28. Zoccolotti, J.O.; Tasso, C.O.; Arbeláez, M.I.A. Properties of an acrylic resin after immersion in antiseptic soaps: Low-cost, easy-access procedure for the prevention of denture stomatitis. PLoS One 2018, 13, https://doi.org/10.1371/journal.pone.0203187.

29. Jaya, S.; Durance, T.D. Influence of processing methods on mechanical and structural characteristics of vacuum microwave dried biopolymer foams. Food Bioprod Process, Trans IChem E: Pt C 2007, 85, 264272, https://doi.org/10.1205/fbp07012. 